Relations industrielles

Industrial Relations

\title{
Human Relations in Industry, par Burlegh Gardner et David Moore, Richard Idwin, Inc., Homewood, 4ième édition, 1964, 479 p.
}

\section{Laurent Bélanger}

Volume 20, numéro 1, 1965

URI : https://id.erudit.org/iderudit/027558ar

DOI : https://doi.org/10.7202/027558ar

Aller au sommaire du numéro

Éditeur(s)

Département des relations industrielles de l'Université Laval

ISSN

0034-379X (imprimé)

1703-8138 (numérique)

Découvrir la revue

Citer ce compte rendu

Bélanger, L. (1965). Compte rendu de [Human Relations in Industry, par Burlegh Gardner et David Moore, Richard Idwin, Inc., Homewood, 4ième édition, 1964, 479 p.] Relations industrielles / Industrial Relations, 20(1), 198-199.

https://doi.org/10.7202/027558ar

Tous droits réservés (C Département des relations industrielles de l'Université Laval, 1965
Ce document est protégé par la loi sur le droit d'auteur. L'utilisation des services d'Érudit (y compris la reproduction) est assujettie à sa politique d'utilisation que vous pouvez consulter en ligne.

https://apropos.erudit.org/fr/usagers/politique-dutilisation/ 
touration de ce nouveou type de supervision. Elle doit foire sienne les notions de « démocratie » (lisez permisiveness in personal interoction), de perfectibilité humaine. Elle doit développer un biais contre lo manipulotion, l'occent mis sur lo hiérachie.

II fout aussi mettre en place de nouvelles structures organisotionnelles où les divisions por fonctions hiérarchiques et non-hiérarchiques doivent disporaître pour faire ploce, en particulier, ò un département qui s'occuperoit du développement des structures et des hommes. La cellule de l'organisation ne serait plus le poste de travail, mais l'équipe de trovoil que constituerait une unité de bose. Les communicotions doivent prendre un caractère clinique. Brièvement, ce serait l'emploi de l'approche * Rogerienne s, lorsqu'il s'agit de conseiller un subordonné ou d'opprécier son rendement.

\section{Formation des cadres: nouvelle vague}

Tout en conservant les méthodes tradionnelles qui mettent l'accent sur l'opproche individuelle en ce qui concerne l'apprentissage et le recyclage, l'approche clinique met 'accent sur l'acquisition, l'aptitude sociale (social skills) par les méthodes utilisées en dynamique de groupe et dans la solution de problèmes en groupe (group-problem solving). Voici quelques-uns des ingrédients que comporte la formation dans l'optique a Clinique 2 :

1 - two-way communications

2-a listening type of interaction

j-consultation

4 - coaching

5 -. Therapeutic onalysis of personnel problem cases

6...T The exercice of firm command where the situation requires it.

\section{Une brève critique :}

$1 /$ est difficile de ne pas admirer le désir des auteur d'implanter ce type c nouveau de supervision, mois il ne fout pas pour autont passer sous silence les faiblesses de cet cuvrcge tont sur le plan théorique que sur le plon de la présentation. Sur le plan théorique, l'ambition des auteurs frôle l'utopie, puisqu'il existe une catégorie de gens qui ne sont pas intéressés à se perfectionner, à prendre des responsabilités additionnelles même si on crée un contexte pour qu'ils puissent le faire.

De plus, les auteurs n'ont jomais réussi ò définir exactement ce qu'on entend par «relations interpersonnelles efficaces
Existe-t-il une méthode pour mesurer l'efi. cocité en relations interpersonnelles?

Lo présentotion du volume n'est pas un succès. Les têtes de chapitre donnent très peu d'indications sur l'enchainement des idées moîtresses et fovorisent d'interminobles répétitions qui en rendent lo lecture oc. coblonte. Cependont, ceux qui sont fomiliers ovec lo littérature en sciences du comportement oppliquées à l'odministration trouveront dans ce volume un réservoir d'idée

Laurent BELANGER

Human Relotions in Industry, por Burlegh Gordner et Dovid Moore, Richard Idwin Inc., Homewood, 4ième edition, 1964, $479^{\circ} \mathrm{p}$

Depuis la première édition du volume de Gordner and Moore, l'intérêt pour l'applica. tion du * sciences du comportement * en administration des entreprises $n^{\prime} a$ cessé de croitre. C'est pourqui les auteurs ont centré leur attention sur le rôle de l'exécutif et la contribution possible qui peuvent lui apporter les connaissances nouvelles en relo. tions humoines dans l'occomplissement efficoce de son rôle. On se souvient que to première édition mettait l'accent surtout sur lo structure de l'entreprise conçue comme un système sociol fermé, coupé des autres types d'organisation sociale, sur la nature de l'interraction oux échelons les plus bos de la pyramide sociale. Tout en retenant beaucoup de l'approche anthropologique, les auteurs font ressortir le comportement et la personnolité des exécutifs en s'attachant ò ses fonctions d'entrepreneurs. A cette fin, il fallait replocer l'entreprise dans l'économie nationale et étudier les relations qu'elle entretient avec d'outres institutions dans un contexte social toujours changeant. Ceci leur a permis d'étudier le rôle de l'exécutif dans ses relations d'échange basée sur la norme de la réciprocité. Le modèle d'échanges est emprunté à Rodcliffe - Brown Parsons et Gouldner.

L'exécutif apparait comme un grand strotège si l'on songe que la stratégie est, en particulier, une ligne de conduite, une norme qui s'est avérée efficace dans une relation d'échange. Nous considérons donc que les chopitres sur les dimensions de l'entreprise d'affaires et les fonctions directoriales s'éloignent des sentiers battus pour apporter une perspective nouvelle en odministration

Cependont, nous croyons que les auteurs sont un peu optimistes en nous recommendont ce volume pour les étudiants qui commencent des études en administration. 
II faudrait une introduction qui permette de retracer le profil de l'entrepreneur troditionnel, l'entrepreneur actuel et le chef d'entreprise salarié. De plus, il foudrait être fomilier ovec l'approche structuro-fonctionnelle, les textes de Gouldner et Homans sur le madèle naturel et rationnel de l'organisation sociale, enfin ovec les textes qui discutent des normes de réciprocité.

\section{Lourent BELANGER}

* The Crisis in the American Trade-Union Movement», Numéro spécial de The Annals of the Americon Acodemy of Political and Social Science. Vol. 350, November 1963, Philodelphia. 230 pp.

A différentes intervalles, cette revue de houte teneur consacre un numéro spécial à l'étude des problèmes du syndicolisme. En 1936, elle avait publié \& Problems of Organized Labor et en 1951, "Labor in the American Economy $\gg$. Cette fois, faisant oppel à des spéciolistes de renom, elle étudie The Crisis in the American TradeUnion Movement ». Ces collaborateurs ont travaillé sous la direction de Solomon Barkin et d'Albert Blum.

Tout d'abord on se pose a question ò sovoir si vroiment il existe une telle crise. Edward $T$. Townsend soutient l'affirmative et compare cette crise d̀ celle des annnées trente. Pour Philip Toft, cela est loin d'être aussi évident : les changements occupationnels et l'importance croissonte des professionnels et des cols blancs ne sont pas des obstocles sérieux à lo prospérité future du syidicalisme. Cependant les dirigeants ouviers eux mêmes, selon une enquête, sont inquiets. Ils attribuent la situation octuelle à des facteurs internes et externes au syndicolisme: changements technologiques, chômage, législation défavorable, flaiblesse dans les structures et les tactiques syndicales, ocroissement du pouvoir patronal et hostilité de l'opinion publique.

Partant de I'hypothèse de l'existence d'une crise, les trois aspects suivants sont envisagés par plusieurs outeurs: lo crise dans les structures; lo crise dans la réalisation des objectifs; les voies de l'ovenir.

Cette crise n'est cependant pas exclusive au mouvement ouvrier américain : on la rencontre cussi dans tous les autres pays. En guise de conclusion, Solomon Barkin suggère lo créotion d'une commission nationale des solutions

Nous considérons que The Annals ont rendu un grond service en levont le voile sur un problème qui intéresse non seulement les dirigeants syndicoux mais tous ceux qui ont à coeur le maintien et le développement de nos institutions démocratiques.

\section{Gérard DION}

\section{Les Groupes sociaux, forces vives? par Henr} Théry. Collection \& Faits sociaux, faits numains $》$. Institut culture et promotion. Editions du Centurion 17, rue de Babylone Poris, 7. 1964. 222 pages.

« Nos institutions et nos représentations de la vie sociale sont encore marquées par des modèles anciens où la société ne se concevait que sous la forme d'un monolithe. Tout s'y ramenait ò un type de socialité unique : celui de la cité, ou celui de la famille, plus exactement de la communauté domestico-fomiliole, ou encore celui de la notion. L'idée que les types de socialité puissent être distincts les uns des autres tout en s'interpénétrant, comme l'idée que la vie personnelle et la vie collective puissent se présenter à la fois comme inséparables et comme irréductibles, n'effleurait guère ceux qui pensaient ou qui organisaient le monde. Dès lors la seule méthode possible pour eux était celle de l'identification.. La réalité présente nous oblige à abandonner ces représentations monolithiques et abstraites.

Dans cet ouvrage l'auteur nous invite à réfléchir sur le phénomène des groupements. II anolyse leurs rapports avec la personne et lo société globale. II s'attarde à étudier le probème de la participotion, de l'intégration dans la vie locale et la vie politique. Excellent pédagogue, l'auteur part de l'expérience que chacun a pu acquérir; puis à l'aide du résultat des recherches sociologiques il amène graduellement le lecteur à rectifier ses jugement, à réformer ses attitudes.

L'auteur, qui est secrétaire général des Semaines sociales de France avait déjà publié des études remarquables sur le sujet, particulièrement dans Crise du pouvoir et crise du civisme (1954). Socialisation et personne humaine (1960) et La société démocratique (1963). II les reprend ici et les intègre.

Au moment où, un peu partout, on prend conscience du rôle que doivent jouer les " corps intermédiaires», cet ouvrage qui réussit à faire le pont entre la vulgarisation et le savoir scientifique sera un instrument très précieux entre les mains de tous ceux 